PROCEEDINGS OF THE AMERICAN MATHEMATICAL SOCIETY

Volume 125, Number 3, March 1997, Pages 861-867

S 0002-9939(97)03655-1

\title{
FUNCTIONS OPERATING FROM A COMPLEX BANACH SPACE TO ITS REAL PART
}

\author{
EGGERT BRIEM
}

(Communicated by Theodore W. Gamelin)

\begin{abstract}
We consider functions operating from a complex Banach function space to its real part. We show among other things, that if $|b| \in \operatorname{Re} B$ for all $b$ in an ultraseparating Banach function space $B$, then $\operatorname{Re} B=C_{\mathbf{R}}(X)$.
\end{abstract}

\section{INTRODUCTION}

Let $B$ be a Banach space of real- or complex-valued continuous functions on a compact Hausdorff space $X$. A continuous function $h$ defined on an interval of the real line or in a domain of the complex plane is said to operate on $B$ if the composition $h \circ b$ belongs to $B$ whenever it is defined, i.e. whenever $b(X)$ is contained in the domain of definition for $h$.

Quite a number of papers have dealt with operating functions for Banach spaces of continuous functions (e.g. [1], [2], [4], [8], [7], [5] and [6]). In one of these papers [5], the author deals with a related case: Let $B$ be a complex Banach function algebra and $h$ a real-valued function defined in a domain in the complex plane such that $h \circ b \in \operatorname{Re} B$, the space of real parts of functions in $B$, whenever the composition of $h$ with $b$ is defined. What can be said about $B$ or $h$ ? More generally one can replace complex Banach function algebras by complex Banach function spaces. Operating functions for real Banach spaces is a special case because if $h$ operates on a real Banach function space $B$, then the function $h_{1}(s+i t)=h(s)$ operates from $B_{1}=B+i B$ to $\operatorname{Re} B_{1}=B$.

In [5] O. Hatori shows that if $B$ is a function algebra and $B \neq C(X)$, then only harmonic functions can operate from $B$ to $\operatorname{Re} B$. For general Banach function algebras, such as the algebra of continuously differentiable complex-valued functions on the unit interval with the norm given by $\|f\|=\|f\|_{\infty}+\left\|f^{\prime}\right\|_{\infty}$, the class of functions operating from the algebra to its real part is much larger.

In the process of characterizing operating functions for a function algebra one shows that if a non-harmonic function operates, then the function algebra must be a Dirichlet algebra. Now, a Dirichlet algebra on a compact Hausdorff space $X$ is ultraseparating on $X$ (definition below) and this property, first studied by A. Bernard [1], is crucial in the proofs. For ultraseparating Banach function algebras the result is the same as for function algebras (see [5]).

Received by the editors March 9, 1995 and, in revised form, October 4, 1995.

1991 Mathematics Subject Classification. Primary 46E15, 46J10.

Key words and phrases. Operating functions, function space, ultraseparation.

(c)1997 American Mathematical Society 
In this note we study the more general case when $B$ is a Banach function space. We show that there exists an ultraseparating Banach function space $B$, with $\operatorname{Re} B \neq$ $C_{\mathbf{R}}(X)$, such that the function $h(z)=|z|^{2}$ operates from $B$ to $\operatorname{Re} B$. Otherwise the results are similar to the ones in the algebra case, some a bit weaker though.

We prove that if $B$ is an ultraseparating Banach function space on $X$, for which there is a continuous function $h$ operating from $B$ to $\operatorname{Re} B$ which is neither harmonic nor of the type $h(z)=|z|^{2}$ on any open set, then $\operatorname{Re} B=C_{\mathbf{R}}(X)$. Thus if $h(z)=$ $|z|^{p}$, where $p \neq 2$, operates from $B$ to $\operatorname{Re} B$, then $\operatorname{Re} B=C_{\mathbf{R}}(X)$. In particular, if the function $h(z)=|z|$ operates from $B$ to $\operatorname{Re} B$, then $\operatorname{Re} B=C_{\mathbf{R}}(X)$. This contrasts with the real case where there is an example of an ultraseparating Banach function space which is not all of $C_{\mathbf{R}}(X)$, but for which the function $h(t)=|t|$ operates. (See [5].) We also give an example of a Banach function space, which is not all of $C(X)$, whose real part equals $C_{\mathbf{R}}(X)$.

\section{The MAIN RESUlts}

In what follows, unless otherwise stated, $B$ will denote a complex Banach function space on a compact Hausdorff space $X$. This means that $B$ separates the points of $X$, contains the constant functions, and that the norm $\|\cdot\|$ on $B$ dominates the sup-norm $\|\cdot\|_{\infty}$. Then $\operatorname{Re} B$, the space of real parts of functions in $B$, is a real Banach function space on $X$ in the norm $\|u\|=\inf \{\|b\|: b \in B$ and $\operatorname{Re} b=u\}$. (We use the same symbol for the norm on $\operatorname{Re} B$ as for the norm on $B$.)

When trying to show that only functions of a special kind can operate on a Banach function space or, from a Banach function space to its real part, one tries to show that the Banach function space in question equals $C(X)$, if it has an operating function not of this special kind. The main tool is Bernard's Lemma. The space $l^{\infty}(B)$ of all bounded sequences of functions from $B$ is a subspace of $l^{\infty}(C(X))$, the space of all bounded sequences of continuous functions on $X$. Bernard's Lemma says that if $l^{\infty}(B)$ is dense in $l^{\infty}(C(X))$, then $B=C(X)$.

Now, the space $l^{\infty}(C(X))$ can be realized in a natural way as the space of all continuous functions on a compact Hausdorff space, namely the space $\beta(\mathbf{N} \times X)$, the Stone-Čech compactification of $\mathbf{N} \times X$. The correspondance is given by $\left\{f_{n}\right\}(k, x)=$ $f_{k}(x)$ for $\left\{f_{n}\right\} \in l^{\infty}(C(X))$ and $(k, x) \in \mathbf{N} \times X$. Thus, a necessary condition for $l^{\infty}(B)$ to be dense in $l^{\infty}(C(X))$, is that $l^{\infty}(B)$ separates the points of $\beta(\mathbf{N} \times X)$. When that condition is satisfied we say that $B$ is ultraseparating on $X$. If e.g. $B$ is a Dirichlet algebra on $X$, then $B$ and $\operatorname{Re} B$ are ultraseparating on $X$, (see [1]). There are also intrinsic characterizations of the ultraseparation property. We will state and make use of one of those later on.

We begin with a result for subspaces, not necessarily ultraseparating, of $C_{\mathbf{R}}(X)$.

Theorem 2.1. Let $B$ be a subspace of $C(X)$ containing the constant functions and let $h$ be a continuous real-valued function defined on the open unit disc $U$ of the complex plane, such that $h \circ b \in \overline{\operatorname{Re} B}$, the closure of $\operatorname{Re} B$ in $C(X)$, for all $b \in B$ for which $b(X) \subset U$. If $h$ is neither harmonic nor of the type $h(s+i t)=$ $\alpha\left(s^{2}+t^{2}\right)+\beta s+\gamma t+\delta$, then $\operatorname{Re} B$ is dense in $C_{\mathbf{R}}(X)$.

Proof. Let us first suppose that $h$ is a $C^{\infty}$-function in $U_{\delta}=\{z \in \mathbf{C}:|z|<\delta\}$ where $0<\delta<1$. Let $u, v \in B$ with $\|u\|_{\infty}<\delta$ and $\|v\|_{\infty}<1$ and define for each $\xi \in \mathbf{R}$ with $|\xi|<\delta-\|u\|_{\infty}$ an element $k(\xi)$ of $\overline{\operatorname{Re} B}$ by putting $k(\xi)=h \circ(u+\xi v)$. 
Differentiating twice w.r.t. $\xi$ and then putting $\xi=0$ we deduce that

$$
\left(h_{s s} \circ u\right) v_{1}^{2}+2\left(h_{s t} \circ u\right) v_{1} v_{2}+\left(h_{t t} \circ u\right) v_{2}^{2} \in \overline{\operatorname{Re} B}
$$

where the indices on $h$ denote partial derivatives and where $v_{1}$ and $v_{2}$ are the real and imaginary parts of $v$. Replacing $v$ by $i v$ and by $v+i v$ we find that

$$
\left(h_{s s} \circ u\right) v_{2}^{2}-2\left(h_{s t} \circ u\right) v_{1} v_{2}+\left(h_{t t} \circ u\right) v_{1}^{2} \in \overline{\operatorname{Re} B}
$$

and that

$$
\left(h_{s s} \circ u\right)\left(v_{1}-v_{2}\right)^{2}+2\left(h_{s t} \circ u\right)\left(v_{1}^{2}-v_{2}^{2}\right)+\left(h_{t t} \circ u\right)\left(v_{1}+v_{2}\right)^{2} \in \overline{\operatorname{Re} B} .
$$

Adding (1) and (2) we deduce that

$$
(\Delta h \circ u)\left(v_{1}^{2}+v_{2}^{2}\right) \in \overline{\operatorname{Re} B}
$$

and subtracting (2) from (1) that

$$
\left(h_{s s} \circ u-h_{t t} \circ u\right)\left(v_{1}^{2}-v_{2}^{2}\right)+4\left(h_{s t} \circ u\right) s v_{1} v_{2} \in \overline{\operatorname{Re} B} .
$$

Also, subtracting (1) and (2) from (3) gives

$$
\left(h_{t t} \circ u-h_{s s} \circ u\right) v_{1} v_{2}+\left(h_{s t} \circ u\right)\left(v_{1}^{2}-v_{2}^{2}\right) \in \overline{\operatorname{Re} B} .
$$

Now, let $u$ be a constant function. Multiplying (5) with $h_{s s} \circ u-h_{t t} \circ u$ and (6) with $4\left(h_{s t} \circ u\right)$ and adding, we find that

$$
\left(\left(h_{s s} \circ u-h_{t t} \circ u\right)^{2}+4\left(h_{s t} \circ u\right)^{2}\right)\left(v_{1}^{2}-v_{2}^{2}\right) \in \overline{\operatorname{Re} B} .
$$

If $u(x)=z$ for all $x \in X,(4)$ and (7) show that

$$
\Delta h(z)\left(v_{1}^{2}+v_{2}^{2}\right) \in \overline{\operatorname{Re} B}
$$

and that

$$
\left(\left(h_{s s}(z)-h_{t t}(z)\right)^{2}+4\left(h_{s t}(z)\right)^{2}\right)\left(v_{1}^{2}-v_{2}^{2}\right) \in \overline{\operatorname{Re} B}
$$

for all $z \in U_{\delta}$ and all $v=v_{1}+i v_{2} \in B$.

We are assuming that $h$ is not harmonic in $U$. Replacing $h$ by $h_{1}(z)=h\left(\alpha z+z_{0}\right)$ for a suitable choice of $\alpha$ and $z_{0}$ we can from the start assume that $h$ is not harmonic in any neighbourhood of 0 . Thus, there is a $z_{1} \in U_{\delta}$ for which $\Delta h\left(z_{1}\right) \neq 0$. Also, using the other assumptions on $h$, we may assume that there is a $z_{2} \in U_{\delta}$ for which $\left(h_{s s}\left(z_{2}\right)-h_{t t}\left(z_{2}\right)\right)^{2}+4\left(h_{s t}\left(z_{2}\right)\right)^{2} \neq 0$. From (8) and (9) it then follows that $v_{1}^{2} \in \overline{\operatorname{Re} B}$ for all $v_{1} \in \operatorname{Re} B$. The Stone-Weierstrass Theorem now implies that $\operatorname{Re} B$ is dense in $C_{\mathbf{R}}(X)$.

For the general case we first note that if $\varphi \in C_{0}^{\infty}(\mathbf{C})$ has support in $U_{\delta}$, then $(h * \varphi)(z)$ is defined for $z \in U_{1-\delta}$. Approximating the integral defining $h * \varphi$ with Riemann sums we deduce that $(h * \varphi) \circ v \in \overline{\operatorname{Re} B}$ if $v(X) \subset U_{1-\delta}$.

If $h$ is not a $C_{0}^{\infty}$-function in any neighbourhood of 0 , we can find a $C_{0}^{\infty}$-function $\varphi$ such that $h * \varphi$ is not harmonic in a neighbourhood of 0 . From the first part of the proof it follows that $v_{1}^{2}+v_{2}^{2} \in \overline{\operatorname{Re} B}$ for all $v=v_{1}+i v_{2} \in B$. We can also find a $C_{0}^{\infty}$-function $\psi$ such that $h * \psi$ is not a polynomial of degree 2 . From the first part of the proof it follows that $v_{1}^{2}-v_{2}^{2} \in \overline{\operatorname{Re} B}$ for all $v=v_{1}+i v_{2} \in B$. We conclude that $v_{1}^{2} \in \overline{\operatorname{Re} B}$ for all $v_{1} \in \operatorname{Re} B$. As in the first part it follows that $\operatorname{Re} B$ is dense in $C_{\mathbf{R}}(X)$.

The next examples show why the restrictions on $h$ in Theorem 2.1 are necessary. 
Example 2.2. Let $A$ be a function algebra on a compact Hausdorff space $X$ and let $h$ be a harmonic function defined in the open unit disc $U$. Then $h$ operates from $A$ to $\operatorname{Re} A$. To see this let $a$ be a function in $A$ for which $a(X) \subset U$. Since $a(X)$ is a compact subset of $U$, we can find a smaller open disc $U_{\delta}$ containing $a(X)$ and a harmonic function $k$ on $U$ such that the restriction of $h+i k$ to $U_{\delta}$ is a bounded analytic function. Then the function $(h+i k) \circ a$ belongs to $A$ and its real part is $h \circ a$.

Example 2.3. Let $B$ be the Banach function space on the unit circle $\Gamma$ of the complex plane spanned by the functions $b(z)=\lambda+\mu z$ where $\lambda$ and $\mu$ are complex numbers, and let $h(s+i t)=s^{2}+t^{2}$. Then

$$
(h \circ b)(s+i t)=|\lambda|^{2}+|\mu|^{2}+2\left(\lambda_{1} \mu_{1}+\lambda_{2} \mu_{2}\right) s+2\left(\lambda_{2} \mu_{1}-\lambda_{1} \mu_{2}\right) t
$$

where $\lambda=\lambda_{1}+i \lambda_{2}$ and where $\mu=\mu_{1}+i \mu_{2}$, which shows that $h \circ b \in \operatorname{Re} B$.

For the next result we impose slightly stronger conditions on the operating function $h$. Before stating that result we need the following characterization of the ultraseparation property:

Proposition 2.4. Let $B$ be an ultraseparating Banach function space on a compact Hausdorff space $X$. Then there exists a natural number $N$ and a positive number $M$, such that for any $f \in C_{\mathbf{R}}(X)$ with $\|f\|_{\infty} \leq 1$, one can find functions $b_{i, k_{i}} \in B_{M}$, the $M$-ball of $B$, where $1 \leq i, k_{i} \leq N$, and numbers $\varepsilon_{i}= \pm 1$, such that

$$
\left\|f-\sum_{i=1}^{N} \varepsilon_{i}\left|b_{i 1}\right|^{2}\left|b_{i 2}\right|^{2} \cdots\left|b_{i k_{i}}\right|^{2}\right\|_{\infty}<1 / 2 .
$$

Proof. If the statement of the proposition is false, then we can find an increasing unbounded sequence $\left\{M_{n}\right\}$ of positive numbers such that there exists for each pair $n, M_{n}$ a function $f_{n} \in C_{\mathbf{R}}(X)$ with $\left\|f_{n}\right\|_{\infty} \leq 1$, such that the condition is not satisfied for $n$ or $M_{n}$.

Let $l^{\infty}\left(|B|^{2}\right)$ denote the linear span of all the sequences $\left\{\left|b_{n}\right|^{2}\right\}$, where $\left\{b_{n}\right\} \in$ $l^{\infty}(B)$. Then $l^{\infty}\left(|B|^{2}\right)$ separates the points of $\beta(\mathbf{N} \times X)$, and thus there is an element $\left\{g_{n}\right\} \in \operatorname{alg}\left(l^{\infty}\left(|B|^{2}\right)\right)$, the algebra generated by $l^{\infty}\left(|B|^{2}\right)$, such that $\left\|\left\{f_{n}\right\}-\left\{g_{n}\right\}\right\|_{\infty}<1 / 2$. Since $\left\{g_{n}\right\}$ is a finite sum of products of elements from $l^{\infty}\left(|B|^{2}\right)$, we have reached a contradiction.

Corollary 2.5. If $B$ is ultraseparating on $X$, then there is a natural number $m$ such that $l^{\infty}\left(|B|^{2}\right)^{m}$ is dense in $l^{\infty}\left(C_{\mathbf{R}}(X)\right)$, where $l^{\infty}\left(|B|^{2}\right)^{m}$ denotes the linear span of all products of at most $m$ elements of $l^{\infty}\left(|B|^{2}\right)$.

Theorem 2.6. Let $B$ be an ultraseparating Banach function space on a compact Hausdorff space $X$ and let $h$ be a continuous function, defined on an open subset $O$ of the complex plane, which operates from $B$ to $\operatorname{Re} B$. Suppose $h$ is neither harmonic nor of the type $h(s+i t)=\alpha\left(s^{2}+t^{2}\right)+\beta s+\gamma t+\delta$ on any open subset of $O$. Then $\operatorname{Re} B=C_{\mathbf{R}}(X)$.

Proof. Pick a function $b_{1}$ in $B$ and a positive number $r$ such that $\left(b_{1}+b\right)(X) \subset O$ if $b$ is in the $r$-ball $B_{r}$ of $B$. Now,

$$
b_{1}+B_{r}=\bigcup_{n}\left\{b \in B_{r}:\left\|h \circ\left(b_{1}+b\right)\right\| \leq n\right\} .
$$


By the Baire Category Theorem there are a function $b_{0} \in B$ and positive numbers $\epsilon$ and $M$ such that

$$
\left\|h \circ\left(b_{0}+b\right)\right\| \leq M
$$

for all $b$ in some dense subset of the $\epsilon$-ball $B_{\epsilon}$ of $B$. Hence

$$
h \circ\left\{b_{0}+b_{n}\right\} \in \overline{l^{\infty}(\operatorname{Re} B)},
$$

the closure of $l^{\infty}(\operatorname{Re} B)$ in $C(\beta(\mathbf{N} \times X))$, if $b_{n} \in B_{\epsilon}$ for all $n$.

The proof now splits into two parts, depending on whether $h$ is a $C^{\infty}$-function or not.

Part 1. Let $V$ be an open subset of $O$ such that $h$ is a $C^{\infty}$-function on $V$, and let $\lambda \in V$ be chosen such that $\Delta h(\lambda) \neq 0$. We let $b_{1} \equiv \lambda$ and choose a smaller $r$ if necessary to ensure that $\lambda+b(X) \subset V$ for all $b$ in $B_{r}$.

We now proceed as in the proof of Theorem 2.1. Differentiating $h \circ\left\{b_{0}+\xi b_{n}\right\}$ we deduce that

$$
\left(\left\{u_{n}\right\}^{2}+\left\{v_{n}\right\}^{2}\right)\left(\Delta h \circ b_{0}\right) \in \overline{l^{\infty}(\operatorname{Re} B)}
$$

for all $\left\{b_{n}\right\}=\left\{u_{n}\right\}+i\left\{v_{n}\right\} \in l^{\infty}(B)$.

If $r$ tends to 0 , then $b_{0}$ tends to $b_{1}$ so that by (11),

$$
\left(\left\{u_{n}\right\}^{2}+\left\{v_{n}\right\}^{2}\right) \Delta h(\lambda) \in \overline{l^{\infty}(\operatorname{Re} B)},
$$

and hence

$$
\left\{u_{n}\right\}^{2}+\left\{v_{n}\right\}^{2} \in \overline{l^{\infty}(\operatorname{Re} B)}
$$

for all $\left\{b_{n}\right\}=\left\{u_{n}\right\}+i\left\{v_{n}\right\} \in l^{\infty}(B)$.

The conditions on $h$ imply that there is another $\lambda \in V$ for which

$$
\left(h_{s s}(\lambda)-h_{t t}(\lambda)\right)^{2}+4\left(h_{s t}(\lambda)\right)^{2} \neq 0 .
$$

We differentiate $h \circ\left\{b_{0}+\xi b_{n}\right\}$, proceed as in the proof of Theorem 2.1 and then let $b_{0}$ tend to $b_{1}$, and find that

$$
\left(h_{s s}(\lambda)-h_{t t}(\lambda)\right)\left(\left\{u_{n}\right\}^{2}-\left\{v_{n}\right\}^{2}\right)+4 h_{s t}(\lambda)\left\{u_{n}\right\}\left\{v_{n}\right\} \in \overline{l^{\infty}(\operatorname{Re} B)}
$$

and that

$$
\left(h_{t t}(\lambda)-h_{s s}(\lambda)\right)\left\{u_{n}\right\}\left\{v_{n}\right\}+h_{s t}(\lambda)\left(\left\{u_{n}\right\}^{2}-\left\{v_{n}\right\}^{2}\right) \in \overline{l^{\infty}(\operatorname{Re} B)}
$$

for all $\left\{b_{n}\right\}=\left\{u_{n}\right\}+i\left\{v_{n}\right\} \in l^{\infty}(B)$.

From (13) and (14) we deduce that

$$
\left\{u_{n}\right\}^{2}-\left\{v_{n}\right\}^{2} \in \overline{l^{\infty}(\operatorname{Re} B)}
$$

for all $\left\{b_{n}\right\}=\left\{u_{n}\right\}+i\left\{v_{n}\right\} \in l^{\infty}(B)$.

It follows from (12) and (15) that $\left\{\operatorname{Re} b_{n}\right\}^{2} \in \overline{l^{\infty}(\operatorname{Re} B)}$ for all $\left\{b_{n}\right\} \in l^{\infty}(B)$. We apply the Stone-Weierstrass Theorem to deduce that $l^{\infty}(\operatorname{Re} B)$ is dense in $l^{\infty}\left(C_{\mathbf{R}}(X)\right)$. Bernard's Lemma now implies that $\operatorname{Re} B=C_{\mathbf{R}}(X)$.

Part 2. Now we are supposing that $h$ is not a $C^{\infty}$-function on any open subset of $O$. If $\varphi$ is a $C_{0}^{\infty}$-function on $\mathbf{C}$ with support in a disc with center 0 and radius $\varepsilon / 2$, then it follows from (10) that

$$
h_{\varphi} \circ\left\{b_{0}+b_{n}\right\} \in \overline{l^{\infty}(\operatorname{Re} B)}
$$

if $b_{n} \in B_{\varepsilon / 2}$ for all $n$, where $h_{\varphi}=h * \varphi$. 
Let $x_{0} \in X$ and put $\lambda=b_{0}\left(x_{0}\right)$. Since $h$ is not a $C^{\infty}$-function in any neighbourhood of $\lambda$, we can find $\varphi$ as above and $\lambda_{1} \in \mathbf{C}$ with $\left|\lambda-\lambda_{1}\right|<\varepsilon / 2$ such that $\Delta^{m} h_{\varphi}\left(\lambda_{1}\right) \neq 0$, where $m$ is chosen using Proposition 2.4 so that $l^{\infty}\left(|B|^{2}\right)^{m}$ is dense in $l^{\infty}\left(C_{\mathbf{R}}(X)\right)$.

Differentiating $h_{\varphi} \circ\left\{b_{0}+\left(\lambda_{1}-\lambda\right)+\xi b_{n}+\eta v_{n}\right\}$ repeatedly we deduce that

$$
\left|b_{1 n}\right|^{2} \cdots\left|b_{m n}\right|^{2} \Delta^{m} h_{\varphi} \circ\left\{b_{0}+\lambda_{1}-\lambda\right\} \in \overline{l^{\infty}(\operatorname{Re} B)}
$$

for all $\left\{b_{1 n}\right\}, \ldots,\left\{b_{m n}\right\} \in l^{\infty}(B)$ and hence

$$
l^{\infty}\left(C_{\mathbf{R}}(X)\right) \Delta^{m} h_{\varphi} \circ\left\{b_{0}+\lambda_{1}-\lambda\right\} \subseteq \overline{l^{\infty}(\operatorname{Re} B)} .
$$

Let $f=\Delta^{m} h_{\varphi} \circ\left(b_{0}+\lambda_{1}-\lambda\right)$. The inclusion above shows that

$$
l^{\infty}\left(C_{\mathbf{R}}(X)\right)\{f\}^{2} \subseteq l^{\infty}\left(C_{\mathbf{R}}(X)\right)\{f\} \subseteq \overline{l^{\infty}(\operatorname{Re} B)} .
$$

Since $\lambda=b_{0}\left(x_{0}\right)$, it follows that $f\left(x_{0}\right)=\Delta^{m} h_{\varphi}\left(\lambda_{1}\right) \neq 0$ and hence $f(x) \neq 0$ for $x$ in a neighbourhood of $x_{0}$. Since $X$ is compact, we can choose finitely many such functions $f$ such that if $g$ is the sum of the squares of these functions, then $g$ is strictly positive on $X$. From the above inclusion we deduce that

$$
l^{\infty}\left(C_{\mathbf{R}}(X)\right)\{g\} \subseteq \overline{l^{\infty}(\operatorname{Re} B)} .
$$

Since $\{g\}>0$ on $\beta(\mathbf{N} \times X)$, it follows that $l^{\infty}(\operatorname{Re} B)$ is dense in $l^{\infty}\left(C_{\mathbf{R}}(X)\right)$ and thus by Bernard's Lemma, $\operatorname{Re} B=C_{\mathbf{R}}(X)$.

Corollary 2.7. If $h$, defined in an open subset $O$ of the complex plane, operates from $B$ to $R e B$ and if $\Delta h$ is not constant on any open subset of $O$, in particular if $h$ is not differentiable on any open subset of $O$, then $R e B=C_{\mathbf{R}}(X)$.

As an example of functions satisfying the conditions of the corollary above, and hence of Theorem 2.6, we mention the functions $h(z)=|z|^{p}$, where $p>0$ and $p \neq 2$. In particular $p=1$ gives the following result:

Corollary 2.8. If $h(z)=|z|$ operates from $B$ to $\operatorname{Re} B$, then $\operatorname{Re} B=C_{\mathbf{R}}(X)$.

As we said in the introduction, there are Banach function spaces $B \neq C(X)$ for which $\operatorname{Re} B=C_{\mathbf{R}}(X)$.

Example 2.9. Let $X=[0,1] \cup[2,3]$ and let $\tau: X \rightarrow X$ be the homomorphism

$$
\tau(x)= \begin{cases}2+t & \text { if } t \in[0,1], \\ t-2 & \text { if } t \in[2,3] .\end{cases}
$$

Let $T: C_{\mathbf{R}}(X) \rightarrow C_{\mathbf{R}}(X)$ be the map

$$
(T f)(t)= \begin{cases}f(\tau(t)) & \text { if } t \in[0,1], \\ -f(\tau(t)) & \text { if } t \in[2,3] .\end{cases}
$$

Now put

$$
B=\left\{f+i T f+\lambda: f \in C_{\mathbf{R}}(X) \text { and } \lambda \in C\right\} .
$$

Since $T^{2}=-I$, the space $B$ is a complex subspace of $C(X)$. It is clear that $B$ is uniformly closed. 
We can ask what happens if we impose on $h$ the weaker conditions from Theorem 2.1. Then we can no longer conclude that $\operatorname{Re} B=C_{\mathbf{R}}(X)$. As mentioned earlier, there is an example by $\mathrm{O}$. Hatori of an ultraseparating real Banach function space $B \neq C_{\mathbf{R}}(X)$ such that the function $h(t)=|t|$ operates from $B$ to $B$. If we put $B_{1}=B+i B$ and $h(s+i t)=|s|$, then $h$ satisfies the conditions from Theorem 2.1 and operates from $B_{1}$ to $\operatorname{Re} B_{1}$, but $\operatorname{Re} B_{1}=B \neq C_{\mathbf{R}}(X)$.

The best result we have obtained for the case when $h$ satisfies the conditions of Theorem 2.1 is that, except for finitely many points of $X, \operatorname{Re} B$ is locally a $C_{\mathbf{R}}(K)$-space, meaning that every $x$ not in some finite subset of $X$ has a compact neighbourhood $K_{x}$ for which $\operatorname{Re} B \mid K_{x}=C_{\mathbf{R}}\left(K_{x}\right)$. This result corresponds to a similar result for operating functions on an ultraseparating real Banach function space (see [3]). One might ask whether in this case the stronger conclusion holds, that $\operatorname{Re} B$ contains every continuous function vanishing in a neighbourhood of some finite subset of $X$. This result, if true, would also correspond to a similar result for real Banach function spaces (see [4]).

\section{REFERENCES}

[1] A. Bernard, Espaces des parties réelles des éléments d'une algébre de Banach de fonctions, J. Funct. Anal., 10 (1972), 387-409. MR 49:7781

[2] A. Bernard, Une fonction non Lipschitzienne peut-elle opérer sur un espace de Banach de fonctions non trivial?, J. Funct. Anal. 122 (1994), 451-477. MR 95h:46036

[3] E. Briem, Operating functions and ultraseparating function spaces, Lecture Notes in Pure and Appl. Math., vol. 136, Marcel Dekker, New York, Basel and Hong Kong, 1991, pp 55-59. MR 93a: 46040

[4] E. Briem and K. Jarosz, Operating functions for Banach function spaces, to appear in the Rocky Mount. J. of Math.

[5] O. Hatori, Range transformations on a Banach function algebra III, Kitasato J. Liberal Arts and Sciences 23 (1989), 78-84.

[6] O. Hatori, Separation properties and operating functions on a space of continuous functions, Int. J. Math. 4 (1993), 551-600. MR 94h:46036

[7] K. de Leeuw and Y. Katznelson, Functions that operate on non-selfadjoint algebras, J. Analyse Math. 11 (1963), 207-219. MR 28:1508

[8] S. J. Sidney, Functions which operate on the real part of a uniform algebra, Pacific J. Math. 80 (1979), 265-272. MR 81b:46069

Science Institute, University of Iceland, Dunhaga 3, 107 Reykjavik, Iceland

E-mail address: briem@rhi.hi.is 